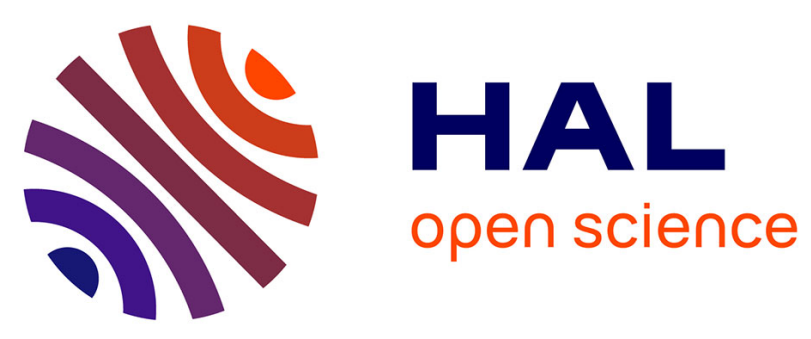

\title{
Socio-économie de proximité
}

Michel Grossetti, Olivier Bouba-Olga

\section{To cite this version:}

Michel Grossetti, Olivier Bouba-Olga. Socio-économie de proximité. Cinquièmes Journées de la Proximité, Jun 2006, Bordeaux, France. hal-00174145

\section{HAL Id: hal-00174145 \\ https://hal.science/hal-00174145}

Submitted on 21 Sep 2007

HAL is a multi-disciplinary open access archive for the deposit and dissemination of scientific research documents, whether they are published or not. The documents may come from teaching and research institutions in France or abroad, or from public or private research centers.
L'archive ouverte pluridisciplinaire HAL, est destinée au dépôt et à la diffusion de documents scientifiques de niveau recherche, publiés ou non, émanant des établissements d'enseignement et de recherche français ou étrangers, des laboratoires publics ou privés. 


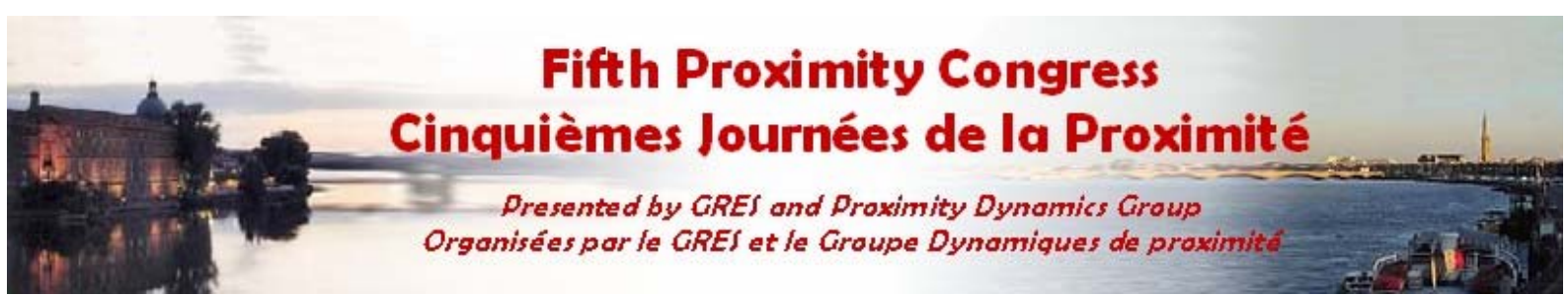

\title{
La proximité, entre interactions et institutions
}

Bordeaux, 28-30 Juin 2006

\section{Socio-économie de proximité}

\author{
Olivier BOUBA-OLGA \\ CRIEF-TEIR, Université de Poitiers \\ obouba@univ-poitiers.fr
}

\author{
Michel GROSSETTI \\ CERS, Université de Toulouse le Mirail \\ Michel.Grossetti@univ-tlse2.fr
}

\begin{abstract}
Résumé : En 1993, le numéro spécial de la Revue d’Economie Régionale et Urbaine consacré aux économies de proximité fondait une approche originale de la dimension spatiale des phénomènes économiques, en même temps qu'il donnait une première expression aux travaux du groupe « Dynamiques de proximité ». Le postulat de base des travaux de ce groupe est de dissocier clairement la proximité dans l'espace physique (à l'échelle des activités humaines) d'autres formes de proximité, constituées par l'activité socio-économique, et a priori distinctes de la proximité physique. A partir de cette distinction essentielle, les membres du groupe ont produit de nombreuses typologies des proximités, tentant de donner à leurs travaux un cadre conceptuel plus précis et partagé.

L'objectif de cet article est de proposer une nouvelle typologie des formes de proximité, qui s'inscrit dans la continuité de celle proposée dans Pecqueur et Zimmermann (2004), mais qui s'en distingue cependant afin d'enrichir l'analyse des travaux récents de la sociologie économique relatifs aux réseaux sociaux. Nous proposons dans un premier temps d'opérer une distinction de base entre une proximité spatiale et une proximité socioéconomique. Dans un deuxième temps, la proximité socio-économique est décomposée en une proximité de ressources et une proximité de coordination. Cette dernière forme de proximité, enfin, est décomposée en une proximité relationnelle (rôle des réseaux sociaux) et une proximité de médiation (rôle des institutions). Sur la base de cette décomposition, nous insistons ensuite sur les interdépendances entre les formes de proximité et sur l'importance des processus d'encastrement et de découplage.
\end{abstract}




\section{Introduction}

En 1993, le numéro spécial de la Revue d'Economie Régionale et Urbaine consacré aux économies de proximité fondait une approche originale de la dimension spatiale des phénomènes économiques, en même temps qu'il donnait une première expression aux travaux du groupe « Dynamiques de proximité ».

Le postulat de base des travaux de ce groupe est de dissocier clairement la proximité dans l'espace physique (à l'échelle des activités humaines) d'autres formes de proximité, constituées par l'activité socio-économique, et a priori distinctes de la proximité physique. A partir de cette distinction essentielle, les membres du groupe ont produit de nombreuses typologies des proximités, tentant de donner à leurs travaux un cadre conceptuel plus précis et partagé.

Sans revenir en détail sur les très nombreuses variantes proposées par les uns ou les autres à des périodes différentes, on peut résumer le mouvement d'ensemble en trois étapes, celle du foisonnement, celle du resserrement, et celle de l'approfondissement.

Dans la première étape, les auteurs ont été amenés à définir beaucoup de formes différentes de proximité. Dans la deuxième étape, un souci de lisibilité les a porté à restreindre à trois puis à deux les formes canoniques de proximité. C’est ainsi qu’après avoir défendu le triptyque «proximité spatiale » - "proximité institutionnelle » - " proximité organisationnelle », les membres du groupe sont revenus à une distinction essentielle entre ce qui relève d'une proximité physique et ce qui relève d'une proximité organisationnelle, la première forme de proximité traitant « de la séparation dans l'espace et des liens en termes de distance », la deuxième « de la séparation économique dans l'espace et des liens en termes d'organisation de la production »(Gilly et Torre, 2000, p. 12-13).

Il s'est avéré que cette distinction fondatrice ne pouvait suffire. Il a donc fallu réintroduire des types différents de proximité, mais non plus dans une typologie « horizontale » comme dans la première étape, mais dans une démarche « verticale » de différenciation successive (de segmentation si l'on préfère) par addition de critères hiérarchisés. C’est ainsi que, s’agissant de la proximité organisationnelle, Gilly et Torre (2000) distinguent deux types de logique qualifiées de similitude et d'appartenance. La logique d'appartenance regroupe des acteurs entre lesquels se nouent des interactions ; la logique de similitude regroupe des acteurs qui se ressemblent, qui possèdent le même espace de référence, partagent les mêmes savoirs, « si bien que la dimension institutionnelle est alors importante » (p. 12). Cette même distinction se retrouve dans un texte récent d'Alain Rallet et André Torre (2005). Mais d'autres auteurs ont 
décomposé autrement la proximité organisée. Ainsi Bernard Pecqueur et Jean-Benoît Zimmermann (2004) distinguent au sein de la proximité organisée les processus de coordination qui sont fondés sur une interaction directe entre les agents — on parle alors de proximité « organisationnelle » — et ceux sans interaction directe — qui renvoient à une proximité « institutionnelle ». On retrouve donc le triptyque défendu il y a quelques années, mais il est ici inséré dans une typologie par segmentation : d'abord distinction entre proximité «spatiale » et proximité « organisée », puis distinction à l’intérieur du second type. Les mêmes auteurs divisent ensuite la proximité « institutionnelle », en distinguant les institutions qui s’imposent aux acteurs (normes) et celles qui doivent leur existence aux agents concernés eux-mêmes (standards). Cette proximité institutionnelle peut également être «inintentionnelle » (logique d'appartenance) ou intentionnelle (logique d’adhésion).

A notre sens, le travail effectué par l'ensemble du groupe est marqué par une progression vers plus de lisibilité, de rigueur et d’opérationnalisation. En particulier, le passage d’une typologie « plate » à une typologie par segmentation nous semble un progrès très important. Il reste cependant de nombreux problèmes. Ainsi par exemple, des confusions apparaissent souvent entre la qualification des liens entre les acteurs et la qualification des acteurs eux-mêmes. C’est notamment le cas lorsque Pecqueur - Zimmermann distinguent une proximité organisationnelle au sens strict (échanges entre acteurs au sein d’une organisation) et la proximité relationnelle (échanges entre acteurs d'organisations différentes). C'est aussi le cas dans le texte de Rallet - Torre lorsqu’ils affirment que la proximité « organisée » est « d'essence relationnelle », ce qui suppose à notre sens que l'on pourrait lui associer des indicateurs de relations entre les acteurs, mais divisent ensuite celle-ci entre une logique de similitude (les acteurs se ressemblent, mais on ne voit pas où sont les relations) ou d'appartenance (idem).

Nous pourrions reprendre ainsi une par une toutes les notions définies par les auteurs du groupe et vérifier leur cohérence et la possibilité de les opérationnaliser. Certaines résisteraient bien à cet examen, d'autres laisseraient vite apparaître leurs limites. Nous avons opté pour une autre démarche, qui consiste à proposer une série d'ajustements, gardant une bonne part des principes et des notions qui nous semblent pouvoir aboutir à des mesures, et introduisant certaines nouveautés destinées pour l'essentiel à clarifier et opérationnaliser les notions. Évidemment, ces changements ne sont pas opérés par des auteurs neutres et impartiaux. Ils reflètent nos propres convictions théoriques. Toutefois, nous avons eu le souci de toujours viser à la fois la cumulativité, la mesure et l’opérationnalisation. 


\section{Retour sur la distinction fondatrice}

Dans un premier temps, il est nécessaire de revenir à la distinction fondatrice du groupe entre proximité d'essence spatiale et proximité d'essence non spatiale. Pour qualifier la première, le groupe utilise le plus souvent le terme de «géographique », après avoir hésité avec le terme «physique », mais en utilisant aussi parfois le terme «spatial». Chaque désignation a ses avantages et ses inconvénients. «Géographique » est probablement le plus juste puisqu’il se réfère à l’espace terrestre (géo) partagé et aménagé par les hommes, mais il pose des problèmes dans les échanges avec les géographes, qui voient dans cette dénomination une réduction de la portée de leur travail. «Physique » pourrait faire croire que l’on ne s’intéresse qu'aux distances purement physiques, sans tenir compte des infrastructures, des coûts de transports, etc. «Spatial » est tautologique puisque toute proximité appliquée à des entités définit ipso facto un espace au sens mathématique du terme. Par commodité, nous utilisons «spatial » dans la suite du texte, mais « géographique » ou « physique » feraient tout aussi bien l’affaire.

En termes d'indicateurs et de mesures, cette proximité peut être évaluée par des distances physiques, des temps de transport, des coûts de transport, des coûts de communication. Dans certains cas, la mesure peut être fondée sur une topologie (on définit un seuil en dessous duquel on est proche, au-delà duquel on n'est pas proche), ce seuil peut être défini a priori (appartenance à une même région) où être déduit (distance en dessous de laquelle des interactions de face à face sont possibles quotidiennement).

Schématiquement, on peut représenter les entités analysées et la proximité spatiale comme suit (dans un espace à une dimension, pour simplifier) :

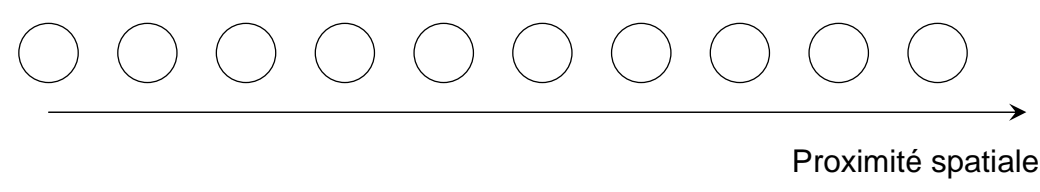

\section{Figure 1 : proximité spatiale}

La proximité d'essence non spatiale est qualifiée d'organisationnelle chez Gilly - Torre puis d’organisée chez Pecqueur - Zimmermann et chez Rallet - Torre : « Par proximité organisée, on entend la capacité qu'offre une organisation de faire interagir ses membres. L'organisation facilite les interactions en son sein, en tous cas, les rend a priori plus faciles qu'avec des unités situées à l'extérieur de l’organisation. » (PAGE ???), une note précisant que « Organisation » est ici un terme générique qui désigne tout ensemble structuré de relations sans préjuger de la forme de la structure. Ce peut être une entreprise, une administration, 
un réseau social, une communauté, un milieu... ». Même si l'on voit bien ce que les auteurs entendent, il est clair que le terme « organisée » suggère l'existence d'une organisation et d'organisateurs, ce qui peut être gênant lorsqu'on l'applique à « tout ce qui n'est pas spatial ». On peut garder le terme « organisé », en faisant référence à l'enchevêtrement des formes socio-économiques qui structurent les échanges et les activités. Il nous semble que le terme initial de Perroux (« distance économique ») était plus clair pour le non initié, à ceci près que le terme « économique » peut sembler trop restrictif. Nous utilisons donc dans ce texte le terme de proximité « socio-économique ».

Nous maintenons donc la distinction fondatrice, en adoptant provisoirement d'autres dénominations qui nous semblent un peu meilleures (surtout pour la seconde), mais en considérant que la question des dénominations est secondaire. Le lecteur est donc laissé libre dans la suite du texte de substituer mentalement " géographique » ou «physique » à « spatiale » et « organisée » à « socio-économique ».

On sait cependant que la proximité socio-économique (ou organisée) est trop englobante. On doit donc la décomposer analytiquement pour en déduire des indicateurs et proposer des mesures adaptées. C’est là que nous proposons quelques ajustements.

\section{La prise en compte préalable des niveaux d'action}

Avant d'opérer cette décomposition nous pensons nécessaire de procéder à une distinction des niveaux d'action. Dès lors, en effet, que l'on étudie des entités collectives (des entreprises par exemple), il convient de s'interroger sur le niveau auquel on se situe pour développer l'analyse. Première possibilité, le niveau individuel : l’organisation est alors définie comme un collectif d'acteurs individuels hétérogènes en interaction, aux objectifs potentiellement divergents. En procédant de la sorte, toutefois, on élimine l'épaisseur des organisations, et donc, dans le même temps, des éléments essentiels participant à leur dynamique. On ne peut plus repérer par exemple l'existence de compétences organisationnelles super-additives, c’està-dire supérieures à la somme des compétences individuelles. 
Une deuxième possibilité consiste à s’affranchir de l'analyse des relations individuelles en se situant au niveau de l'organisation dans son ensemble. Chaque organisation est assimilée à une « boîte noire », l'analyse se concentre sur les relations inter-organisationnelles. Mais cette fois, ce sont les apports de la nouvelle sociologie économique que l’on évacue : on sait par exemple que la construction de certaines relations inter-organisationnelles résulte de relations sociales inter-individuelles antérieures (construction sociale du « marché »).

Pour éviter les biais inhérents à chacune des deux approches, nous proposons de prendre en compte, de distinguer et d'articuler les différents niveaux d'action, en nous limitant (par souci de simplification ${ }^{2}$ ) aux niveaux individuel et collectif. En procédant de la sorte, il nous sera possible, ensuite, d'étudier certains éléments essentiels de la dynamique des organisations (processus d'encastrement et de découplage notamment) qui sont à la base du passage d'un niveau d'action à l'autre, passage qui peut s'accompagner, dans certains cas, de variations dans l'échelle spatiale des organisations.

Ajustement 1 : on considère donc que la prise en compte et la distinction des niveaux individuel et collectif est indispensable pour dérouler des analyses plus pertinentes.

\section{Une nouvelle décomposition de la proximité socio- économique}

Situons-nous d'abord au niveau des individus : on peut distinguer deux façons d'évaluer ce qui rapproche deux individus. La première façon se fonde sur l'analyse de leurs caractéristiques individuelles, indépendamment des possibilités de coordination dont ils disposent : on évaluera alors la mesure dans laquelle ils se ressemblent ou sont complémentaires, du point de vue de leurs activités ou des ressources dont ils disposent. La seconde façon d’évaluer ce qui rapproche les individus consiste à analyser les facilités ou obstacles à leur coordination, indépendamment des caractéristiques individuelles : on centrera alors l'analyse sur les réseaux ou les dispositifs qui structurent la coordination.

Pour distinguer ces deux types de situations, nous proposons les notions de proximité de ressources d'une part, et de proximité de coordination d'autre part.

\footnotetext{
${ }^{2}$ Rien n’empêche d'envisager d'autres niveaux : une troisième possibilité consiste à se focaliser sur des collectifs intermédiaires. Si l'on prend l'exemple de la société de capitaux, on peut ainsi distinguer le collectif des actionnaires, le collectif des dirigeants et le collectif des autres salariés.
} 


\section{Ajustement 2: Décomposition de la proximité socio-économique en deux sous-}

catégories : proximité de ressources et proximité de coordination.

\subsection{Deux formes de la proximité de ressources}

Il y a donc une proximité de ressources. Que sont les ressources ? Précisons d'abord que toute ressource est aussi une contrainte, par les limitations qu'elle donne à l'action. Elle est aussi un enjeu, puisqu'elle fait l'objet de recherches d'appropriation par les acteurs. Les mêmes ingrédients peuvent donc être constitués en ressource (s’ils sont mobilisés activement), en contrainte (s’ils sont subis passivement) ou en enjeu (s'ils font l'objet de luttes pour leur appropriation). Dans le monde social, pratiquement tout peut être constitué en ressource par les acteurs, y compris d'autres acteurs (les « ressources humaines »). Il nous paraît toutefois essentiel de distinguer deux types de ressources (il s'agit ici d’idéaux types, toujours plus ou moins mêlés dans la réalité) : les ressources cognitives (que l’on suppose situées dans la tête des acteurs) et les ressources matérielles. Cela permet de définir deux types de proximité de ressources :

Proximité cognitive : elle renvoie à l'idée d’une similarité ou complémentarité des valeurs, des «allant de soi », des projets, des routines, des conventions, des référents, etc. (toutes choses que l'on peut rassembler sous le terme de « ressources cognitives »). Cette proximité concerne ce qui se passe dans la tête des gens et qui se manifeste par des actions et des discours. La plupart des ressources cognitives sont partageables (la langue, les valeurs, les normes, etc.) et sont mobilisables pour se coordonner. Nous les retrouverons plus sous cet angle. Mais dans la proximité de ressources cognitives, nous nous centrons sur leur statut de ressource individuelle, sur la possibilité de les comparer à ce titre là, et de construire ainsi une évaluation des similarités ou complémentarités qu’elles induisent entre individus.

La proximité cognitive peut parfois se conjuguer à une proximité spatiale. C'est ce que l'on observe dans les districts industriels ou les milieux innovateurs. Le fait que l'on soit proche en termes cognitifs facilitera - argument souvent repris dans la littérature — la circulation des connaissances. Dans d'autre cas, la proximité cognitive ne se conjugue pas à une proximité spatiale. Deux entreprises d'un même secteur mais localisées à distance partagent une « culture » sectorielle commune qui facilitera le cas échéant la circulation de connaissances tacites. En termes d'indicateurs et de mesure, cette forme de proximité est sans doute la plus difficile à repérer. La stratégie incontournable, nous semble-t-il, consiste à analyser les discours et les pratiques d'acteurs pour repérer les « allant de soi » communs. 
Proximité matérielle : les individus sont semblables ou complémentaires sous le rapport des ressources dont ils disposent (patrimoines, revenus, diplômes, statuts sociaux, etc.). C’est la proximité sociale classique (Sorokin, Bourdieu). Même si elle a été conçue par opposition à la proximité dans l'espace (le roi et son valet sont plus distants dans l'espace social que deux rois de pays éloignés), il y a des liens entre les deux types de proximité, qui se retrouvent dans les contrastes socio-spatiaux : l'existence de quartiers « de cadres » ou « de relégation » par exemple. Exemple de complémentarité : les riches habitants des résidences fermées de Rio de Janeiro et leurs serviteurs qui résident à proximité.

\section{Ajustement 3 : décomposition de la proximité de ressources en deux types distincts : matérielle, et cognitive.}

La proximité de ressources peut être fondée sur la similarité (les individus ont des caractéristiques semblables) ou la complémentarité (ils sont complémentaires) ${ }^{3}$. Chacune des proximités qui suit peut donc être déclinée sur ces deux modes.

S’agissant de la proximité de ressources, la schématisation pourrait être la suivante :

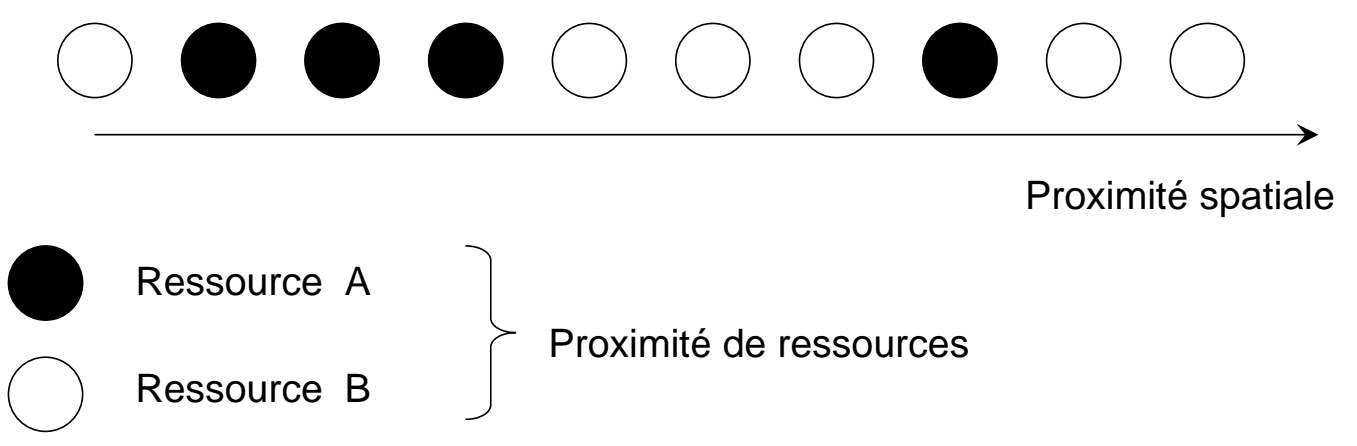

Figure 2 : proximité spatiale et proximité de ressources

Cette distinction est proche de celle entre logique d'appartenance et logique de similitude de Gilly - Torre (ou Rallet - Torre). Fondamentalement, nous gardons l’idée de la logique de similitude avec la proximité de ressources. Par contre, la proximité relationnelle n’est pas une logique d'appartenance. Ici, il faut ouvrir une petite discussion pour bien préciser notre point de vue. Les acteurs sont insérés dans de multiples groupes, organisés ou non, et ils entretiennent des relations. Groupes et relations sont différents (Grossetti et Bès, 2003). Le fait d'appartenir à une organisation (l’Éducation Nationale par exemple) n’implique en rien

\footnotetext{
${ }^{3}$ On pourrait fonder sur cette distinction une subdivision des proximités de ressources, mais nous avons préféré arrêter la prolifération des types de proximité à un niveau cognitivement acceptable...
} 
l'existence d'une relation directe entre deux acteurs. Réciproquement, une relation peut être référée à plusieurs groupes (on milite au même parti et on travaille ensemble) ou à aucun. C'est pourquoi, associer le qualificatif de « relationnel » à la logique «d'appartenance » comme le font Rallet - Torre, nous semble opérer une confusion gênante entre les deux types de « structure sociale ».

\subsection{Deux formes de la proximité de coordination}

Une façon de conserver l'idée de Rallet et Torre en évitant la confusion que nous venons d'évoquer est d'opérer une distinction entre deux formes fondamentales de coordination. La première de ces formes de coordination est constituée par les réseaux sociaux : la structure formée par l'agrégation des relations personnelles influe sur les échanges et les possibilités de coordination. On retrouve là tous les apports de la sociologie économique sur la place des réseaux dans l'action économique. La position des différents acteurs dans les réseaux définit ce que nous appelons une proximité relationnelle. Mais les acteurs ne se coordonnent pas uniquement par les réseaux sociaux. Ils peuvent aussi s’appuyer sur des dispositifs qui permettent d'échanger sans mobiliser des chaînes relationnelles. C’est ce que nous appelons des ressources de médiation ${ }^{4}$, en reprenant une dénomination issue de la sociologie de l'innovation ${ }^{5}$, et plus particulièrement des travaux de Franck Cochoy sur les médiations marchandes (Cochoy, 2002). On peut illustrer cette distinction par l'exemple classique du marché du travail. Un résultat récurrent des recherches dans ce domaine est qu'une part importante des emplois sont trouvés en passant par des chaînes de relations personnelles. Dans son travail sur une population de cadres de la banlieue de Boston, Granovetter évaluait à 56\% cette proportion (Granovetter, 1974). Dans une analyse secondaire des enquêtes emploi de l'INSEE, Michel Forsé évaluait cette part à 35\% pour un échantillon représentatif de la population française. La proximité relationnelle joue donc un rôle significatif dans l'accès à l'emploi. Mais elle ne fait pas tout. Une autre partie des emplois sont trouvés en passant par des candidatures

\footnotetext{
4 C'est ce que nous pourrions appeler également des institutions, si l'on se situe cette fois dans la lignée des travaux les plus récents de l'économie. Mais cela demanderait une discission plus approfondie des deux terminologies.

${ }^{5}$ Nous désignons par ce terme les travaux initiés par Bruno Latour et développés entre autres par Michel Callon, Antoine Hennion (qui a théorisé la notion de médiation), Madeleine Akrich et différents auteurs membres du Centre de Sociologie de l'Innovation ou proche de cette équipe.
} 
spontanées, des petites annonces, des cabinets de recrutement, etc. Pour pouvoir envoyer une candidature spontanée, il faut connaître un minimum d'informations sur l'entreprise, information qui peut être trouvée par le bouche à oreille (à nouveau la proximité relationnelle), mais aussi dans des annuaires ou dans la presse. Les petites annonces sont gérées par des journaux. Les cabinets de recrutement passent leur temps à mettre en contact des futurs employeurs et employés qui ne sont pas au préalable en relation. Les journaux, les annuaires, les moyens de communication (Internet, le téléphone, la Poste, etc.), les intermédiaires humains des organismes de recrutement et de placement constituent selon nous des ressources de médiation qui, pris ensemble, structurent ce que l'on peut appeler la proximité de médiation. Les normes et standards évoqués par Zimmermann et Pecqueur pour fonder la " proximité institutionnelle » peuvent être considérés comme des ressources de médiation. Plus largement, comme nous l'avons signalé plus haut, une grande partie des ressources cognitives peuvent être considérées comme des ressources de médiation : le langage, les normes sociales, etc. Elles ne sont plus considérées alors au niveau individuel comme nous l'avons fait plus haut dans la discussion sur la proximité cognitive, mais comme des ressources de coordination partagées. Si l'on veut, elles sont considérées d'un point de vue individualiste dans la proximité de ressources et d'un point de vue interactionniste dans la proximité de coordination. La distinction peut paraître subtile, mais elle nous paraît nécessaire. Elle peut s’argumenter aussi sur le fait que même des ressources aussi orientées vers la coordination collective que le langage peuvent fonctionner sur un registre strictement individuel, comme lorsque nous notons une liste de tâches à effectuer sur un bout de papier : nous nous coordonnons avec nousmêmes dans le temps.

En fonction des besoins de l'analyse et de la perspective théorique adoptée, on peut définir une typologie plus ou moins fine des ressources de médiation. L’économie de l'entreprise considère par exemple que la coordination des activités économique peut relever de la firme (vue alors comme une ressources de médiation, ou une institution, centralisé régulé par l'autorité), du marché (ensemble de ressources de médiation décentralisé régulé par les prix) ou de la coopération (dispositif de médiation décentralisé régulé par le plan). Avec comme éléments de différenciation essentiels le caractère centralisé ou décentralisé de la décision, d'une part, et l'épaisseur institutionnelle d'autre part : l'épaisseur institutionnelle du marché est faible, celle de la coopération est plus importante.

Ajustement 4: décomposition de la proximité de coordination en deux souscatégories : la proximité relationnelle et la proximité de médiation. 
Les ressources de médiation ont une aire d'efficience plus ou moins étendue. Ainsi Internet ne couvre-t-il pas la planète et toutes les catégories de population, les journaux ne sont disponibles que dans certains espaces, etc. Certains dispositifs sont très génériques (la langue française, Internet), d’autres sont très spécialisés (tel groupe de discussion électronique entre la cinquantaine de spécialistes d'un domaine). Enfin, certains dispositifs sont constitutifs d'organisations. En effet, une organisation n’existe que si elle est dotée d'un nom, de frontières, et de règles diverses définissant l'appartenance, les relations avec l'extérieur, la coordination interne, etc. Tous ces éléments constituent des ressources de médiation qui ont pour effet de découpler l'organisation par rapport à ses membres et aux relations personnelles qu'ils entretiennent (Grossetti et Bès, 2001). L’organisation apparaît alors comme un ensemble d'acteurs et de ressources de médiations spécifiques à ces acteurs.

Si l’on récapitule les deux formes de proximité de coordination, on aura :

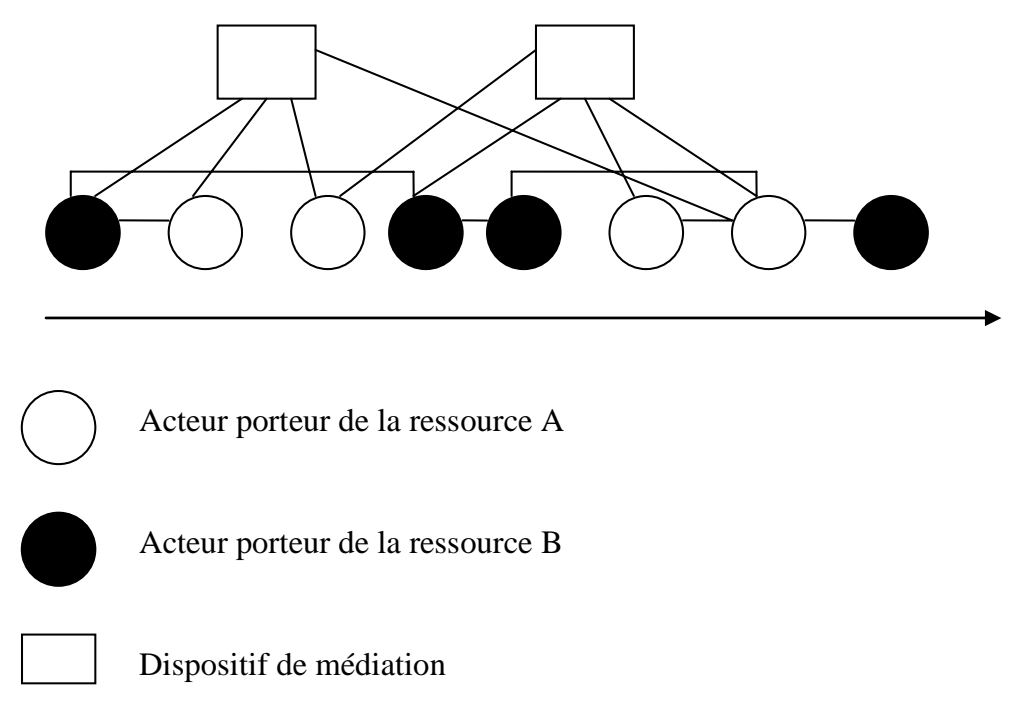

Figure 3 : proximités de coordination

Nous pouvons à présent résumer la typologie obtenue au niveau individuel dans un schéma d'ensemble : 


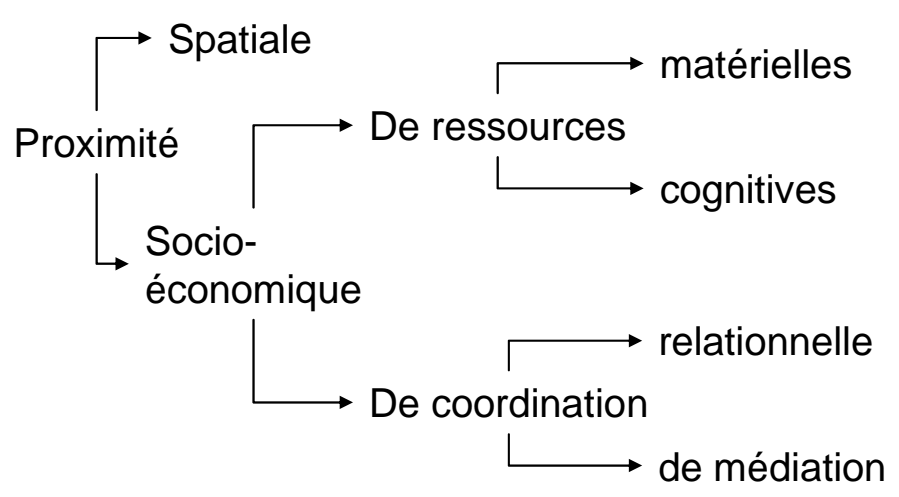

Figure 4 : typologie des formes de proximité

\subsection{Au niveau des organisations}

La typologie des formes de proximité définie au niveau des individus peut-elle être transposée au niveau des organisations?

Commençons par la proximité de ressources. La proximité cognitive n’a de sens pour les organisations que dans la mesure où l'on est en capable d'identifier des formes de mémoire organisationnelle et technique formalisée. Ce n’est pas impossible mais si cela s’avère irréalisable, il vaut mieux laisser de côté cette forme de proximité, qui présente le risque d'un usage purement métaphorique masquant des processus complexes comme l'effet des relations personnelles sur les choix organisationnels. La proximité matérielle est par contre relativement aisée à mettre en œuvre par une analyse des activités (similarité / complémentarité) et des ressources (actifs).

Passons à présent à la proximité de coordination. La proximité relationnelle ne peut se construire qu'en définissant les relations entre organisations. Nous proposons de définir celles-ci comme un engagement formel réciproque (contrat ou convention par exemple). Cet engagement peut prendre des formes variées6, qui sont un équivalent pour les organisations des caractéristiques que l'on a l'habitude d'évaluer pour les relations entre individus (force des liens, polyvalence, etc.). Les relations « informelles » entre organisations renvoient en réalité à des processus d'encastrement qu'il vaut mieux identifier et analyser comme tels, d'autant plus que c'est souvent là que se jouent les effets de proximité spatiale. On peut donc transposer partiellement les notions du niveau individuel au niveau organisationnel, mais il faut adapter tout ce qui est lié à la dimension cognitive : la proximité de même nom et la définition des

\footnotetext{
${ }^{6}$ Par exemple : relation marchande, coopération sur projet, création d’une filiale commune, etc.
} 
relations. La plupart des ressources de médiation sont accessibles à l'échelle des organisations. Là encore, il vaut mieux laisser de côté les aspects les plus cognitifs, difficiles à identifier à ce niveau d'action, et se concentrer sur les dispositifs plus concrets : sociétés de conseil, médias, etc.

On retrouve donc au niveau des organisations les différentes formes de proximité, mais il faut procéder à deux ajustements pour les transposer : définir des relations entre organisations et définir des formes de ressources cognitives organisationnelles (ou laisser de côté cette dimension).

\section{Articulation des niveaux d'analyse et d'action}

Au-delà de cette caractérisation statique des entités et de ce qu'elles partagent, le groupe de proximité insiste largement sur les interdépendances entre les formes de proximité. A ce titre, dans certains cas, la proximité cognitive émerge des interactions entre les acteurs. Elle est le produit de la proximité relationnelle, le processus étant parfois auto-renforçant (c'est la thèse de Storper, 1995).

En allant un cran plus loin, on peut dire que la proximité cognitive, lorsqu'elle découle de la proximité relationnelle, peut ensuite s'en découpler : soit on dira que les conventions partagées par les individus, conventions issues des interactions, se cristallisent dans des institutions (des ressources de médiation dans notre terminologie) et s’appliquent à un ensemble éventuellement plus large d'individus et d'organisations (Storper, ci-dessus) ; soit, dans une optique de sociologie économique, on dira que la proximité cognitive est liée à l'encastrement des individus dans un même réseau social (elle est donc à relier à la proximité relationnelle) mais peut ensuite s’en affranchir (processus de découplage). Le découplage produit des ressources utilisables par les acteurs pour se coordonner, au-delà de leurs relations existantes. Le découplage est toujours associé à la création ou la mobilisation de ressources de médiation qui ont précisément pour effet d’affranchir au moins partiellement les échanges des contraintes de la proximité relationnelle.

Mieux encore, la proximité cognitive peut résulter d'un encastrement social, déboucher sur la mise en place de relations économiques, qui vont se découpler du réseau social initial. Ceci pour dire et insister sur le fait que les relations peuvent être multidimensionnelles, l'apport essentiel de la sociologie économique étant précisément de mettre en évidence cette construction sociale du marché. 
A l'inverse, on peut considérer que, dans certains cas, la proximité relationnelle suppose une proximité cognitive : on ne rentrera en relation (économique par exemple) que si l'on se fait confiance, cette confiance est effective si l'on partage les mêmes valeurs (proximité cognitive).

La dynamisation de l'analyse passe également par la combinaison dans un même schéma explicatif des deux niveaux individuel et organisationnel. On pourra alors mettre en évidence, notamment, les différentes structures de réseau relationnels, certaines observables au niveau des individus, d'autres au niveau des organisations, pour étudier ensuite le passage d'un niveau d'action à un autre. Schématiquement, on aurait, par exemple :

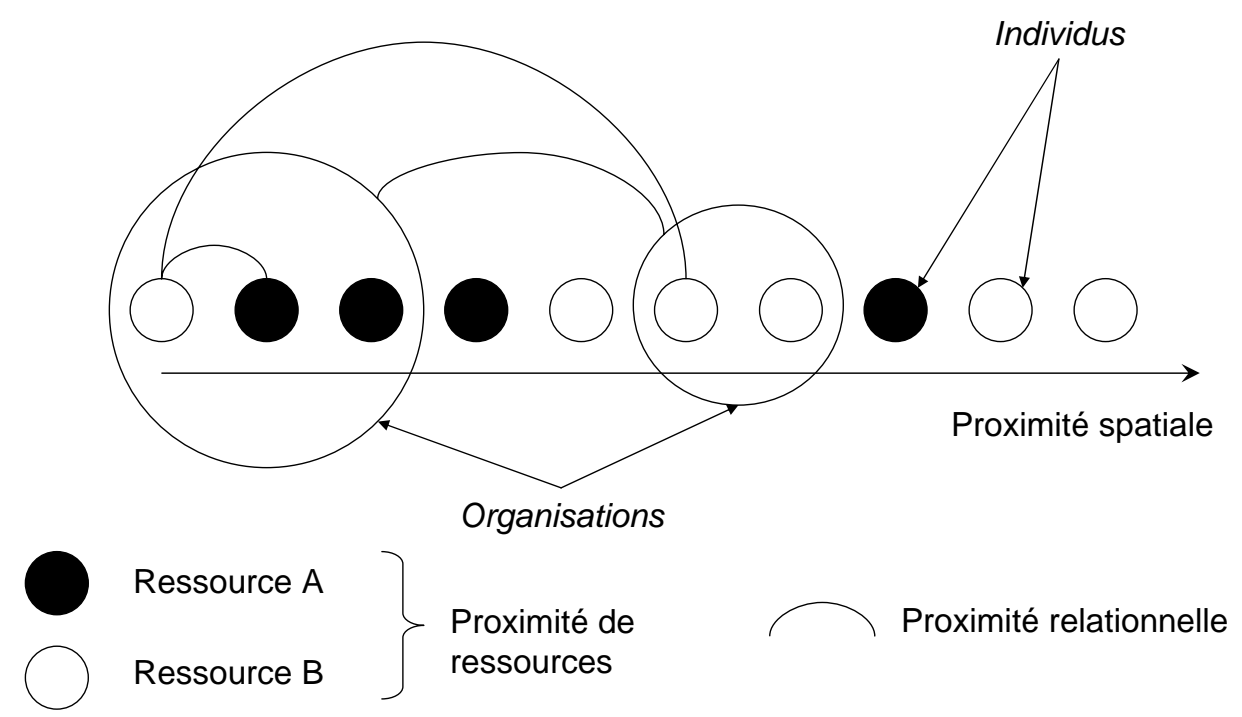

Dans ce schéma, les organisations sont reliées (contrat de R\&D par exemple). Cette proximité relationnelle inter-organisationnelle trouverait son origine dans une proximité relationnelle inter-individuelle : les deux individus considérés sont par exemple issus de la même école d'ingénieurs. Par exemple, lorsque l'organisation A, confrontée à un problème productif (mais parfois, le même mécanisme peut se mettre en marche sans volonté initiale des organisations), a recherché un partenaire, un individu de cette organisation a activé son réseau social, contacté un individu de l'organisation B, et enclenché la relation, rapprochant ainsi les deux organisations modifiant ainsi la proximité relationnelle inter-organisationnelle. On sait qu'ensuite, la relation inter-organisationnelle peut s'affranchir de la relation inter-individuelle (l'un des deux individus quitte l'entreprise, par exemple), on a un processus de découplage. Dans le cas des relations science-industrie, la proximité spatiale des organisations influe sur la proximité spatiale des individus, qui influe sur la proximité relationnelle individuelle, qui favorise les rapprochements entre organisations, à condition que celles-ci soient complémentaires (proximité matérielle à leur niveau). Parfois, mais pas nécessairement, ce mécanisme 
s’accompagne du renforcement de la proximité cognitive par production de normes collectives.

L'interaction entre les effets de proximité à des niveaux d'actions différents fonctionne aussi dans l'autre sens. Par exemple, travailler dans une même organisation implique l'accès aux ressources de médiation spécifiques de ces organisations, sur la base desquels s'effectuent les interactions. Des interactions peuvent naître entre deux membres de l'organisation des spécificités relationnelles (des médiations spécifiques aux deux partenaires), qui font qu’ils deviennent de moins en moins substituables l'un par rapport à l'autre, signe de l'émergence d'une relation sociale, qui se peut se découpler du contexte organisationnel, au point éventuellement de survivre à la disparition de ce contexte (si l'un des protagonistes quitte l’organisation par exemple).

A titre d'exemple de recombinaison des différentes notions, on pourra dire qu'un district est un territoire où se conjuguent proximité spatiale et proximité relationnelle (division localisée du travail), cette proximité relationnelle (relations économiques inter-organisationnelles) s'appuie sur une proximité cognitive et des ressources de médiation qui se sont construits dans le temps long, à partir d'un autre niveau de proximité relationnelle (relations sociales inter-individuelles), elle-même influencée par les collectifs (et les dispositifs qui leur sont spécifiques) dans lesquels les individus exercent ou ont exercé leur activité.

L'un des avantages des définitions proposées est de rendre l'approche de la proximité compatible avec des analyses relevant de l'individualisme, de l'interactionnisme ou du holisme : une analyse holiste insistera par exemple sur le rôle de la proximité cognitive entre les acteurs, proximité cognitive qui renvoie à l'existence d'institutions formelles (lois) et informelles (valeurs partagées). La différenciation territoriale du développement économique s’expliquerait alors par l'existence de profils institutionnels différenciés. Ce type de démarche prévaut dans des travaux régulationnistes récents entrepris à l'échelle internationale : « la complémentarité institutionnelle peut donc expliquer la persistance de modèles différents d'économie capitaliste à partir des interactions entre institutions » (Amable et Petit (2001), souligné par nous). Méthodologiquement, l'analyse de données permet facilement de faire émerger les profils institutionnels des territoires (pays ou régions). L’approche de la proximité pourrait ajouter sa touche en s'interrogeant sur l'étendue spatiale des institutions ainsi repérées. C'est à un tel travail que se livrent par exemple Carrincazeaux et Lung (2005).

Une analyse interactionniste insistera plutôt sur les interdépendances entre proximité relationnelle et proximité cognitive (question de l'émergence des institutions), sur les ressources de 
médiation et sur les allers-retours entre les niveaux inter-individuels et inter-organisationnels (via notamment les processus d'encastrement et de découplage). Une analyse individualiste insistera sur les ressources différentielles que procurent les relations (le « capital social ») ou l’accès aux ressources de médiation.

\section{Conclusion}

Il fallait retravailler la typologie des formes de proximité car celle-ci comportait encore trop de confusions et de pans obscurs. Les typologies produites jusqu'alors avaient entre autres pour limites de mal intégrer les apports de la sociologie économique et de ne pas distinguer les niveaux d'action. Nous avons choisi de construire une nouvelle typologie dans un souci de cumulativité, en considérant que les typologies produites jusqu’à présent s’inscrivaient dans une progression d'ensemble vers une clarification des notions et vers leur opérationnalisation. Notre typologie se veut une étape de plus dans la longue marche du groupe vers la stabilisation d'un cadre théorique clair, robuste et susceptible d'une mise en oeuvre empirique rigoureuse. C'est pourquoi nous avons cherché à modifier le moins possible l'existant, conservant la distinction fondamentale et le principe d'une segmentation en sous-catégories emboîtées. Nous avons modifié des intitulés et en avons proposé de nouveaux, mais nous avons essayé de laisser suffisamment d'ouverture pour revenir éventuellement à certains des intitulés antérieurs. Nous pensons en effet que les idées sont plus importantes que le vocabulaire, même si nous n'en ignorons pas l'importance, et qu'elles peuvent s'exprimer parfois avec des mots différents.

Cette typologie présente à notre sens des avancées très significatives et lève un certain nombre d'ambiguïtés qui pénalisaient jusque là les approches en terme de proximité. Il faudra certainement aller plus loin. Mais pour cela, le meilleur moyen n'est pas à notre sens de raffiner ou de nuancer cette typologie par une démarche théorique, même si cela peut s’avérer nécessaire, c’est plutôt de confronter nos catégories à des terrains variés.

\section{Références citées}

Bouba-Olga O., Zimmermann J.-B., 2004, « Modèles et mesures de la proximité », in Pecqueur B., Zimmermann J.-B. (eds.), Economie de Proximités, Hermès, pp. 89-111.

Carrincazeaux C., Lung Y., 2004, «Configurations régionales des dynamiques d'innovation et performances des régions françaises », Cahiers du GRES, 2004-24. 
Cochoy Franck, 2002, Une sociologie du packaging ou l'âne de Buridan face au marché, Paris, PUF.

Gilly J.-P., Torre A., 2000, Dynamiques de proximité, L’Harmattan.

Granovetter Mark S., 1974, Getting a job. A study of contacts and careers, Harvard University Press.

Grossetti Michel et Bes Marie-Pierre, 2001, “Encastrements et découplages dans les relations science - industrie ”, Revue Française de Sociologie, Vol. 42, n²2, pp.327-355.

Grossetti Michel et Bès Marie-Pierre, 2003, «La dynamique des cercles et des réseaux. Encastrements et découplages », Revue d'économie industrielle, n¹03, pp.43-58.

Grossetti Michel, 2004, Sociologie de l'imprévisible. Dynamiques de l'activité et des formes sociales, Presses Universitaires de France.

Krugman P., 1991, « Increasing returns and economic geography », Journal of Political Economy, Vol. 99, No. 3, pp. 483-499.

Maillat D., 1995, « Milieux innovateurs et dynamique territoriale », in Rallet A., Torre A., 1995, p. 211-231.

Pecqueur B., Zimmermann J.-B. (eds.), 2004, Economie de Proximités, Hermès.

Powell Walter and Smith-Doerr Laurel, 1994, "Networks nd économic life", in Neil J. Smelser and Richard Swedberg (eds), The Handbook of economic sociology, Princeton, Russel Sage Foundation, pp.368-402.

Rallet A., Torre A., 1995, Economie industrielle et économie spatiale, Economica.

Storper M., 1995, « La géographie des conventions : proximité territoriale, interdépendances hors marché et développement économique », in Rallet, Torre, 1995, p. 110-127.

White Harrison C. 2002, Market from networks. Socioeconomic models of production, Princeton University Press, Princeton and Oxford. 